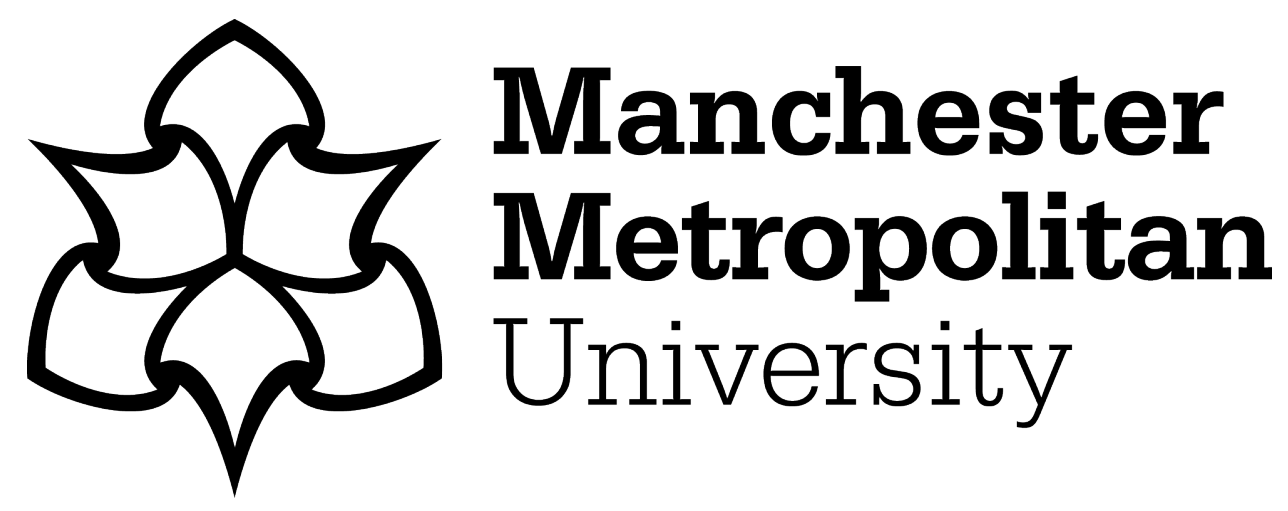

Qureshi, Nawab Muhammad Faseeh, Bashir, Ali Kashif, Siddiqui, Isma Farah, Abbas, Asad, Choi, Keehyun and Shin, Dong Ryeol (2018) A Knowledge-Based Path Optimization Technique for Cognitive Nodes in Smart Grid. In: GLOBECOM 2018 - 2018 IEEE Global Communications Conference, 09 December 2018 - 13 December 2018, Abu Dhabi, United Arab Emirates.

Downloaded from: https://e-space.mmu.ac.uk/622941/

Version: Accepted Version

Publisher: IEEE

DOI: https://doi.org/10.1109/glocom.2018.8648016

Please cite the published version 


\title{
A knowledge-based path optimization technique for cognitive nodes in smart grid
}

\author{
Nawab Muhammad Faseeh Qureshi ${ }^{1 *}$, Ali Kashif Bashir ${ }^{2}$, Isma Farah Siddiqui ${ }^{3}$, Asad Abbas ${ }^{4}$, \\ Keehyun Choi ${ }^{1}$ and Dong Ryeol Shin ${ }^{1}$ \\ ${ }^{1}$ Sungkyunkwan University, South Korea, ${ }^{2}$ University of the Faroe Islands, Denmark \\ ${ }^{3}$ Mehran University of Engineering \& Technology, Pakistan, ${ }^{4}$ Hanyang University ERICA, South Korea
}

\begin{abstract}
The cognitive network uses cognitive processes to record data transmission rate among nodes and applies selflearning methods to trace data load points for finding optimal transmission path in the distributed computing environment. Several industrial systems, e.g., data-centers, smart grids, etc., have adopted this cognitive paradigm and retrieved the least HOP count paths for processing huge datasets with minimum resource consumption. Therefore, this technique works well in transmitting structured data such as 'XML', however, if the data is in unstructured format i.e. 'RDF', the transmission technique wraps it with the same layout of payload and eventually returns inaccuracy in calculating traces of data load points due to the abnormal payload layout. In this paper, we propose a knowledgebased optimal routing path analyzer (RORP) that resolves the transmission wrapping issue of the payload by introducing a novel RDF-aware payload-layout. The proposed analyzer uses the enhanced payload layout to transmit unstructured RDF triples with an append pheromone (footsteps) value through cognitive nodes towards the semantic reservoir. The grid performs analytics and returns least HOP count path for processing huge RDF datasets in the cognitive network. The simulation results show that the proposed approach effectively returns the least HOP count path, enhances network performance by minimizing the resource consumption at each of the cognitive nodes and reduces traffic congestion through knowledge-based HOP count analytics technique in the cognitive environment of the smart grid.
\end{abstract}

Index Terms-Cognitive network, Semantic dataset, Apache Hadoop, RDF Triple, Ant colony optimization, Smart grid.

\section{INTRODUCTION}

A cognitive network is a data communication paradigm that facilitates nodes with runtime on-demand service processing in the distributed computing environment [1]. It's network infrastructure consists of cognitive processes that invoke operations to perform runtime tasks such as record, monitor, append data before it reaches final destination node. Because of these custom feature to fulfill on demand requirements, several organizations adopt cognitive network framework for data processing i.e., IT data-centers and smart grid [2].

A smart grid is a self-driven power generation and distribution system that widely adopts cognitive network features and applies to the infrastructure through three-tier architecture [3]. The first-tier consists of edge nodes that perform

Acknowledgment: This research was supported by Basic Science Research Program through the National Research Foundation of Korea(NRF) funded by the Ministry of Education (NRF-2016R1A6A3A11930831)

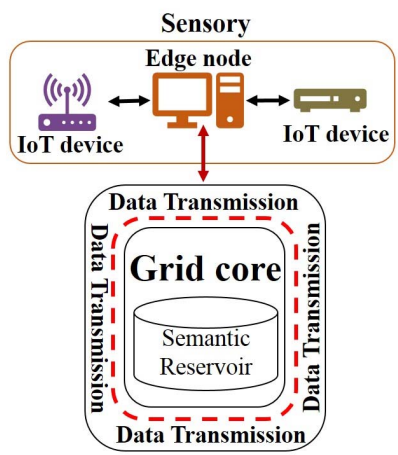

Fig. 1: Smart grid data generation and storage architecture

sensory operations and generate sensor information dataset. The second-tier transforms these datasets into Resource Description Framework (RDF) triples workloads and transmit them through various transmission paths of cognitive network towards semantic repository. The third-tier performs various types of data analytics for maintaining, monitoring and processing activities of smart grid [4] as shown in Fig. 1.

In this way, we observe that the smart grid assess the transmission rate and the distance between network nodes through cognitive processes and draw a routing path to deliver RDF triple towards semantic reservoir [5], [6]. Therefore, the grid finds individual data transmission rate of a node Node $_{T R}=\sum_{\text {Data\&RDFTriple }}^{\text {RDFData }}\left(\frac{\text { nof }}{\text { Bandwidth }}\right)$ and the overall grid nodes transmission rate $\operatorname{Grid}_{T R}=$ $\sum_{\text {DatasetERDFTriple }}^{\text {RDF } \text { fata }_{i}}\left(\frac{\text { RDF }[\text { Packet }]_{i}}{\text { Bandwidth }_{i}}\right)_{n}$ in its cognitive network [7].

These transmissions calculate RDF processing path with HOP count and deliver triples in the semantic reservoir. In this way, we observe that the delivery path only emphasizes on working of cognitive processes and traditional network path optimization strategies to transmit RDF triples. This increases uncertain complexity between cognitive network functionality and measurement of path HOP count and results in a noncompliance among functions in the form of network congestion Flow $_{\text {Node }(i)}=\left(\right.$ TripleChunk $_{k-1} \otimes$ TripleChunk $\left._{k-2}\right)$ [8]. Moreover, it maximizes network overflow issues and deactivates nearest node search function to transmit RDF triples using Euclidean distance $D_{\text {Node }_{i}}=\sqrt{\sum_{j}}\left(x_{j}-x_{i j}\right)^{2}$ [9]. Thus, 


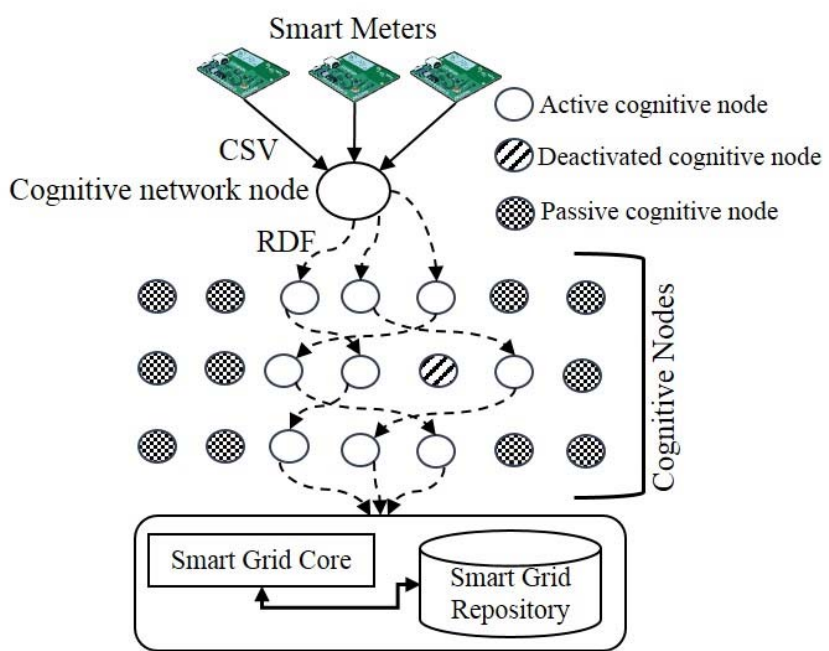

Fig. 2: Default RDF transmission work-flow in smart grid

TABLE I: Smart meter tabular datatypes to RDF datatypes

\begin{tabular}{|l|l|}
\hline Tabular Datatype & RDF datatype IRI \\
\hline Key & xsd:integer \\
\hline SmartMeterID & rdf:langString \\
\hline Location & rdf:langString \\
\hline TimeStamp & xsd:dateTimeStamp \\
\hline Temperature & xsd:float \\
\hline Load & xsd:float \\
\hline
\end{tabular}

grid consumes time latency to locate an alternate of related cognitive nodes and smart grid performs poor in transmitting semantic data towards its reservoir as shown in Fig. 2.

To resolve this issues, we propose RDF-aware optimal routing path strategy (RORP) that analyzes all the transmission paths and extracts least HOP count path through ant colony pheromone technique to transmit RDF dataset into a semantic reservoir. The main contributions of this paper are highlighted as:

- A novel enhancement of payload data segment structure to fix RDF triples.

- A modified cognitive process that reads RDF workload concurrently using customized payload structure.

- Insertion of pheromone value into RDF triple payload.

- Pheromone-aware RDF dataset storage into semantic reservoir.

- A novel knowledge-based RDF data transmission strategy that reduces network congestion and maintains nodes intact to process dataset with least HOP count in the smart grid.

The remaining paper is organized in the following manner. Section II motives the modern technology requirements to adopt ant colony optimization approach for obtaining least HOP count in cognitive network. Section III briefly elaborates RDF-aware optimal routing path strategy (RORP). Section IV presents the performance evaluation of RORP individually and with comparative analysis over designed Apache Hadoop cluster. Section V concludes the proposed approach RORP with recommendations of future works.

\section{Motivation}

Finding optimal routing path with least HOP count in a large-scale cognitive network such as smart grid is no less than a challenge in today's distributed computing environment. There are several schemes and routing protocols that focus to address this issue by effective gradient data transmission schemes [10], adaptive HOP routing protocols [11], robust diffusion algorithms using ant colony optimization [12] and energy-efficient diffusion routing algorithms [13], however, no of them resolves this problem through knowledge-based analytics. To the best of our knowledge, RORP is first of its kind that addresses optimal routing path issue through calculating load onto cognitive network nodes and append load value as pheromone on its RDF-enabled data payload. The semantic reservoir stores this RDF payload and performs analytics over large-scale repository that highlights information related to all the routing paths of smart grid with minute processing details that helps finding optimal path having least HOP count through data analytics.

\section{RDF-AWARE OPtIMAL Routing PATH (RORP)}

The proposed approach (RORP) finds least HOP count path of processing RDF triples in the cognitive network of smart grid. This strategy consists of five steps i.e., A) RDF triple payload, B) knowledge-based cognitive processing, C) append pheromone onto triple payloads, D) pheromone-aware RDF reservoir and E) Knowledge-based optimal routing path analyzer.

RORP uses these steps in such a hierarchical way that, at first, a simple message payload is transformed into RDF triple compatible payload. In the next step, it calculates the individual load on each of the triple payloads using RDFaware cognitive process. After that, a pheromone value is appended onto every triple payload and those pheromoneaware payloads are stored in a semantic reservoir. In the last, the optimal routing path analytics evaluate the minimum HOP count used path in the cognitive network of a smart grid.

\section{A. Step-1: RDF Triple Payload}

The payload is a data part of RDF triple transmitted onto the cognitive network of smart grid. By default, the structure of triple payload consists of a single data block that stores subject, predicate and object information in a single capsule [14] and can be expressed as,

$$
R D F_{\text {Packet }}=\left(\text { Header }_{\text {Length }}, \text { Payload }_{\text {Length }}\right)
$$

RORP enhances this payload packet message into three parts such as sub-payload, pred-payload and obj-payload with individual header id assigned to each of them. Therefore, when a cognitive process performs a network operation, RDF triple returns individual part data transmission rate along with its execution time-stamp. The categorized payload packet is expressed through Fig. 3 and can be obtained as,

$\operatorname{RORP}_{R D F(\text { Packet })}=\left\{\left(H_{\text {Sub }}, P_{\text {Sub }}\right),\left(H_{\text {Pred }}, P_{\text {Pred }}\right),\left(H_{O b j}, P_{O b j}\right)\right\}$ 


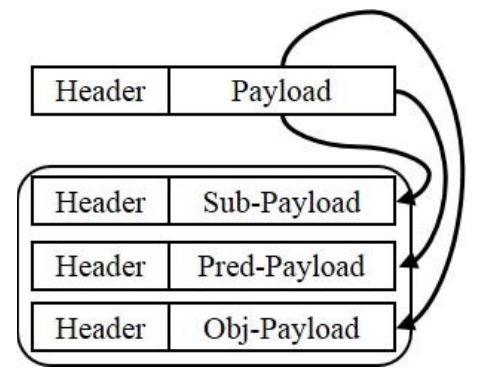

Fig. 3: RORP RDF triples storage payload

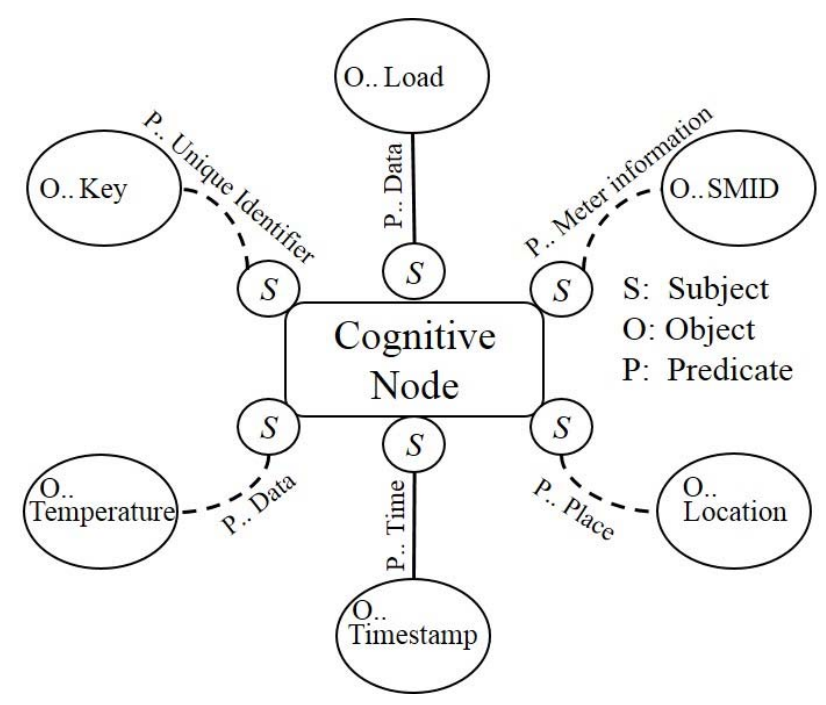

Fig. 4: Cognitive node RDF triple processing

and the transmission rate can be expressed as,

$R O R P_{R D F(\text { Rate })}=\left\{\frac{\left(H_{\text {Sub }}, P_{\text {Sub }}\right)}{T}, \frac{\left(H_{\text {Pred }}, P_{\text {Pred }}\right)}{T}, \frac{\left(H_{O b j}, P_{O b j}\right)}{T}\right\}$

Where, $H_{S u b}, H_{P r e d}$ and $H_{O b j}$ represent the individual subject, predicate and object headers packets and $P_{S u b}, P_{P r e d}$ and $P_{O b j}$ depicts each subject, predicate and object payload packets. The ' $T$ ' is the transmission rate that calculates the duration of sending and receiving of an RDF triple packet.

\section{B. Step-2: Knowledge-based Cognitive Processing}

The knowledge-based cognitive process is a function that analyzes an RDF triple payload $R O R P_{R D F(\text { Packet) }}$ passing through a single node of cognitive network. As we are transmitting RDF payloads of a smart meter [15], so the function assess individual dataset columns such as element key, SMID, load, temperature, location and time-stamp values. The bandwidth used in processing each of the triple subpayload is recorded onto the cognitive network and can be calculated as,

$$
\operatorname{Load}_{C P_{i}}=\frac{\operatorname{RORP}_{R D F(\text { Rate })}}{\text { Bandwidth }_{R D F(\text { Sub }, \text { Pred }, O b j)}}
$$

The cognitive node keeps a records for each of the individual sub-payloads of smart meter passed through it as shown in
Fig. 4. The collective node load of RDF triple payloads can be calculated as,

$$
\operatorname{Load}_{\text {Node }_{i}}=\frac{\sum \operatorname{Load}_{C P_{i}}}{\operatorname{CP}_{(\text {Memory }, C P U, I / O)}}
$$

\section{Step-3: Append Pheromone onto Triple Payloads}

The term 'pheromone' represents a footstep value used to memorize the workload on a network path. In case of a cognitive network, we use cognitive process value $\operatorname{Load}_{C P_{i}}$ as a pheromone that keeps track of previous individual RDF triple processing on a particular node. The pheromone between two cognitive nodes can be expressed as,

$$
P_{\text {Node }(i, j)}=\frac{\left(\tau_{i, j}^{\operatorname{Load}_{C P_{i}}}\right)}{\left(\tau_{i, j}^{\operatorname{Lodd}_{\text {Node }}, j}\right)}
$$

Where, ' $\tau$ ' is the amount of pheromone and ' $P$ ' depicts the obtained value of pheromone in the reservoir of cognitive network. In order to get a complete perspective of pheromone between two nodes, RORP updates it with recent $\operatorname{Load}_{C P_{i}}$ values as,

$$
\tau_{i, j}^{\operatorname{Load}_{C P_{i}}}=(1-p) \tau_{i, j}^{\operatorname{Load}_{C P_{i}}}+\Delta \tau_{i, j}^{\operatorname{Load}_{C P_{i}}}
$$

Where, ' $p$ ' is the rate of pheromone evaporation and ' $\Delta \tau_{i, j}$ ' is the pheromone deposited into semantic reservoir as,

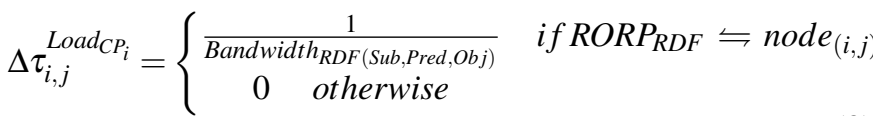

In order to receive pheromone statistics of all the nodes of cognitive network, RORP updates it with recent Load $_{\text {Node }_{i}}$ values as,

$$
\tau_{i, j}^{\text {Load }_{\text {Node }_{i}}} \leftarrow(1-p) \tau_{i, j}^{\text {Load }_{\text {Node }_{i}}}+\sum_{k=1}^{m} \Delta \tau_{i, j}^{\text {Load }_{\text {Node }_{k}}}
$$

Where, $m$ is the number of nodes and $\Delta \tau_{i, j}^{\text {Load }_{\text {Node }_{k}}}$ is the pheromone value over the cognitive network as,

$$
\Delta \tau_{i, j}^{\text {Load }_{\text {Node }_{i}}}=\left\{\begin{array}{l}
\frac{1}{\text { Bandwidth }_{\text {Node }_{i}}} \\
0 \quad \text { otherwise }
\end{array} \text { if RORP }_{\text {RDF }} \leftrightharpoons \text { node }_{(i, j)^{k}}\right.
$$

\section{Step-4: Pheromone-aware RDF Reservoir}

The pheromone-aware RDF reservoir contains a computational environment that includes node table, triple indexes, prefix table and in-line values. The node table manages RORP triple datasets $\tau_{i, j}^{\operatorname{Load}_{C P_{i}}}$ with a customized concatenation declaration of pheromone value with triple payload $R O R P_{R D F(\text { Packet })}$. The triple indexes consists of twodimensional double array accessibility feature that fetches the triple payload with associated pheromone values. The prefix table maps pheromone-index with large-scale node table index and in-line values wrap pheromone and triple data-types together to present a single RDF entity as obtained through stored RORP dataset $\Delta \tau_{i, j}^{\text {Load }_{\text {Node }_{i}}}$. 


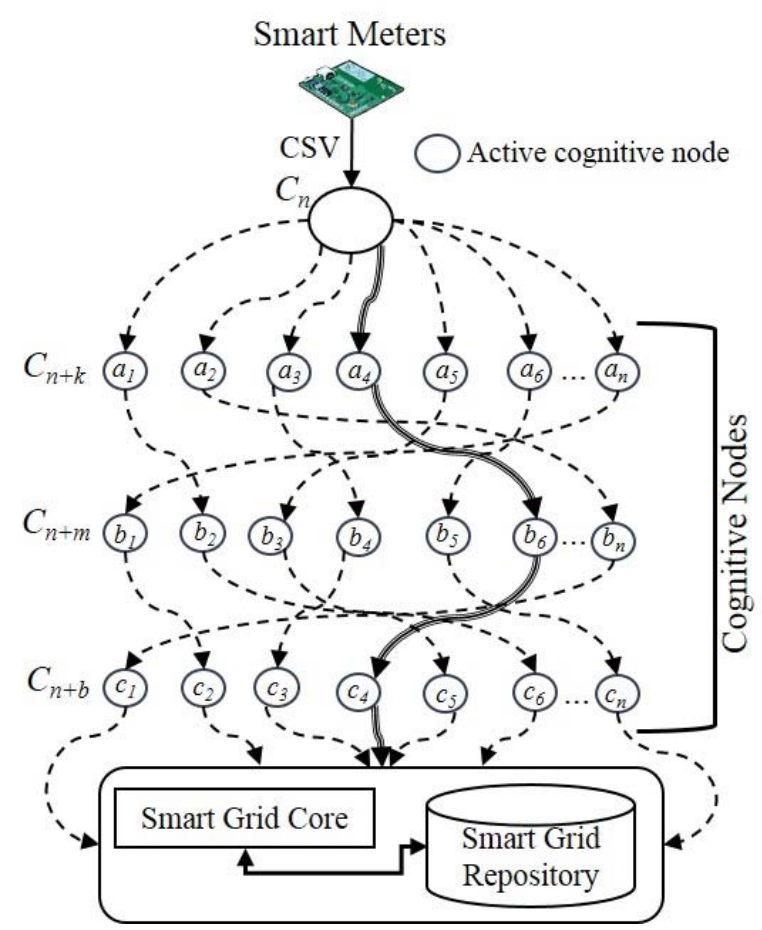

Fig. 5: Knowledge-base optimal path strategy

\section{E. Step-5: Knowledge-based Optimal Routing Path Analyzer}

The optimal routing path analytics is a procedure to identify the shortest path having least HOP count to process RDF triples. This is carried out through processing a knowledgebased analyzer over stored $\Delta \tau_{i, j}^{\text {Load }_{\text {Node }_{i}}}$ pheromone RDF triple dataset on the semantic reservoir of the smart grid as shown in Fig. 5.

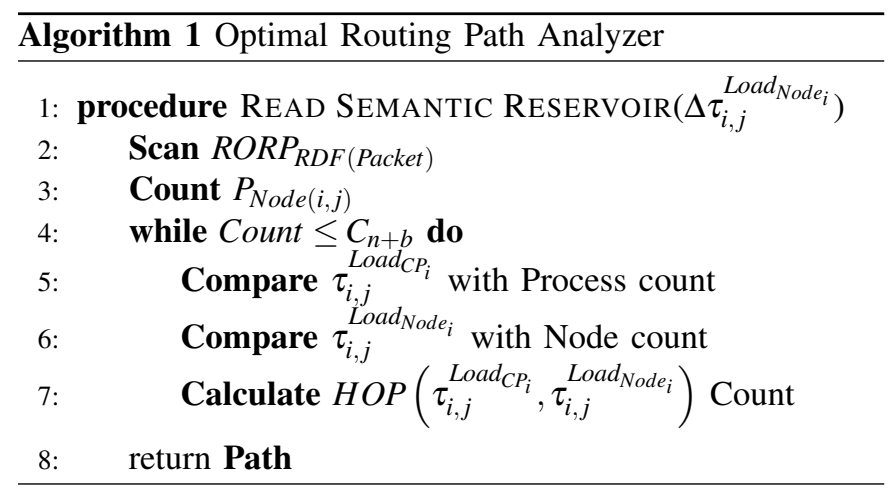

Algorithm-1 presents a systematic retrieval of HOP count through the semantic reservoir pheromone-aware dataset

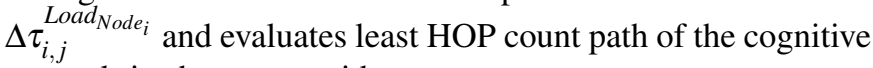
network in the smart grid.

\section{EXPERIMENTAL WORK}

In order to evaluate RORP, we performed experimental executions over a Hadoop cluster configuration as mentioned in Table-II.

\section{A. Environment}

The hardware configuration includes Intel Xeon processor with $8 \mathrm{CPU}$ units, 32GB processing memory and storage media i.e. 128GB Samsung SSD, 1TB disk Seagate Hard Disk Drive, and tmpfs utility for RAM_DISK storage processing. Additionally, we have used Intel core i5 processor with 4 Cores, 16GB processing memory and storage media i.e. 128GB Samsung SSD, 500GB Hard Disk Drive, and temfs utility as RAM_DISK storage processing. The virtual environment includes Virtualbox 5.1.34 over 5 virtual machine configurations as elaborated in Table-III.

\begin{tabular}{|c|c|c|c|}
\hline Machine & Specifications & \multicolumn{2}{|c|}{ No. of VM } \\
\hline Intel Xeon E5-2600 v2 & $\begin{array}{c}\text { 8 CPUs, 32GB memory, 1T } \\
\text { Disk and 128 GB SSD }\end{array}$ & 3 & $\begin{array}{c}\text { 1 Master Node, } \\
\text { 2 Datanodes }\end{array}$ \\
\hline Intel core i5 & $\begin{array}{c}\text { 4 Core, 16GB memory, 1T } \\
\text { Disk and 128 GB SSD }\end{array}$ & 2 & 2 Datanodes \\
\hline Hadoop & Hadoop-2.7.2 (stable) & Apache-Jena-fuseki-3.6.0 \\
\hline $\begin{array}{c}\text { Virtual Machine } \\
\text { Management }\end{array}$ & \multicolumn{3}{|c|}{ VirtualBox 5.0.16 } \\
\hline
\end{tabular}

TABLE II: Hadoop Cluster Configuration

\begin{tabular}{|c|c|c|c|c|}
\hline Node & CPU & Memory & Disk & Configuration \\
\hline Master Node & 6 & $16 \mathrm{~GB}$ & HDD,SSD,RAM & Intel Xeon \\
\hline Slave1 & 2 & $4 \mathrm{~GB}$ & HDD,SSD,RAM & Intel Xeon \\
\hline Slave2 & 2 & $4 \mathrm{~GB}$ & HDD,SSD,RAM & Intel Core i5 \\
\hline Slave3 & 2 & $4 \mathrm{~GB}$ & HDD,SSD,RAM & Intel Core i5 \\
\hline Slave4 & 2 & $4 \mathrm{~GB}$ & HDD,SSD,RAM & Intel Core i5 \\
\hline
\end{tabular}

TABLE III: Virtual Machines in Hadoop Cluster

\section{B. Experimental Dataset}

The dataset used to process experimental work includes:

1) 1 GB CSV (source file) (10 Smart meters unstructured dataset) [16], [17]

\section{Experimental Results}

The experiments conducted to evaluate RORP are:

1) RDF Triple payload storage,

2) Cognitive process pheromone generation,

3) Pheromone-aware RDF Triple,

4) Cognitive node load analytics, and

5) Comparative analysis.

1) RDF Triple Payload Storage: By default, an RDF triple contains a layout of two structure segments such as header and payload. The header stores prefix and postfix acknowledgment messages that identifies a data packet layout and the payload segment manages subject, predicate and object tuple information [14]. The proposed analyzer RORP redesign this payload segment into three sub-parts such as sub-payload, pred-payload and obj-payload each equivalent of $1 \mathrm{~KB}$ to store individual tuple information through 'PAYLOAD_CAT' function. Simultaneously, it extracts triples from source dataset and wrap individual tuple data using a wrapper function 'PAYLOAD_WRAP' for storing into the already generated sub-payloads segments. This reduces RDF 


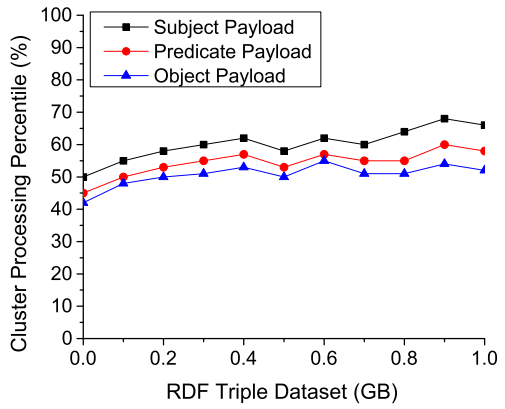

Fig. 6: RDF Sub-payloads Workload in Cognitive Network

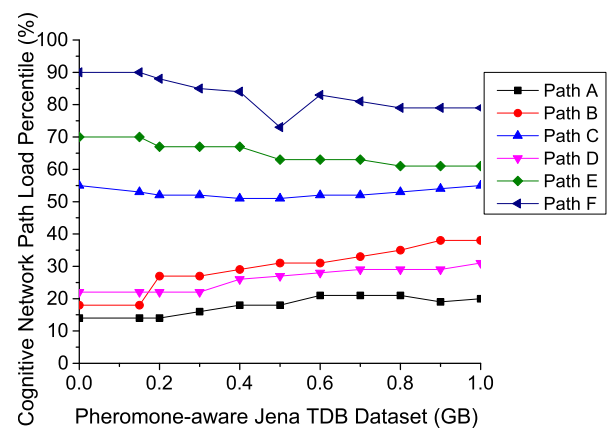

Fig. 9: Pheromone-aware RDF Triple Paths in Cognitive Network

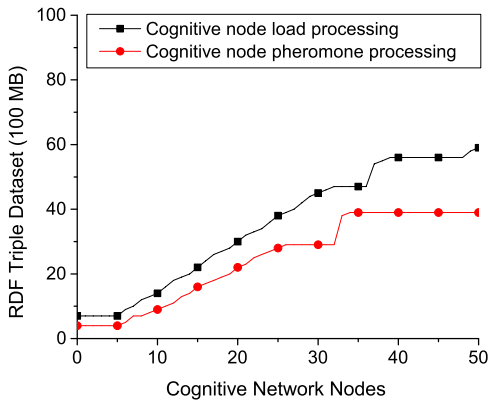

Fig. 7: Performance of Load and Pheromone processing

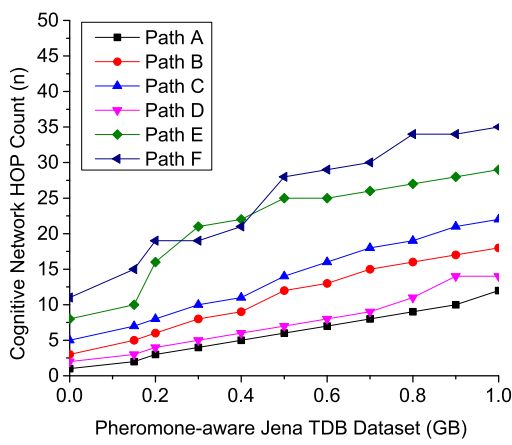

Fig. 10: Pheromone-aware RDF HOP Counts

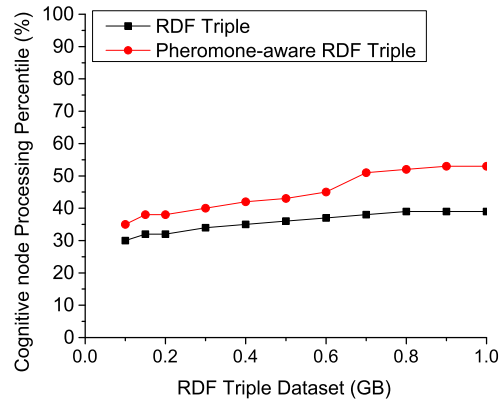

Fig. 8: 'Slave1' Node Pheromone aware and original RDF processing

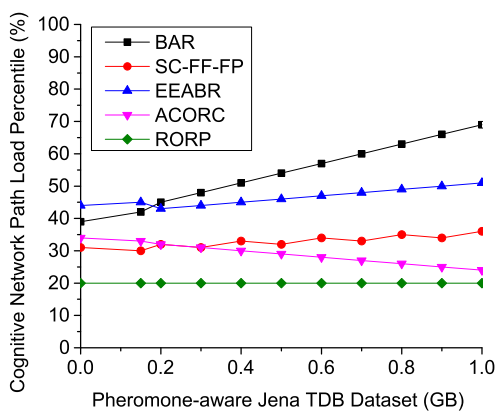

Fig. 11: Path load analysis with multiple path optimizers payload transmission workload evenly on the cognitive nodes and improves the performances of cognitive processes in the smart grid as shown in Fig. 6.

2) Cognitive Process Pheromone Generation: A cognitive process uses TRIPLE_RECORD function to capture RDF triples workload passing through the network nodes. As we know that, the load of a node is measured in terms of memory consumed per RDF triple [2]. Therefore, this load value is labeled with the name of 'pheromone' and identified as the workload footsteps at particular interval of time. RORP observes these footsteps with a time-interval of ' 5 ' seconds and incorporates them within the new 'sub-payloads' of RDF triple. In this way, we observe two individual activities i.e., load processing and pheromone processing. In the load processing, cognitive process records original RDF 'subpayload' sample that consumes computing capacity, memory and I/O of the node, where as the pheromone processing consumes less computing capacity, memory and I/O of the node compared to load processing because of simply encapsulating pheromone sample to existing RDF 'sub-payload' of $100 \mathrm{MB}$ dataset as shown in Fig. 7.

3) Pheromone-aware RDF Triple: Pheromone-aware RDF triple includes the footstep record value along with original sample of RDF. In order to evaluate an actual difference between pheromone-aware and original RDF triple processing, we processed ' 2 ' individual datasets i.e., with-pheromone and without-pheromone on a single node 'slave1' as discussed in Table-III and observed an additional consumption of node processing percentile in pheromone-aware RDF triple case than original RDF triple as shown in Fig. 8. This extra consumption of node processing percentile will no longer be a disadvantage once Hadoop cluster starts performing analytics on semantic reservoir.

4) Cognitive node load analytics: The cognitive network of smart grid performs various cognitive processes simultaneously to transmit RDF triples through multiple paths [18]. The same is the case of pheromone-aware RDF triple processing onto a cognitive network that also adopts various paths to transmit RDF triples towards semantic reservoir. There can be many reasons for adopting multiple paths such as decreasing the factor of failure in RDF transmission, reducing the assumption of triple corruption and ensuring the storage of exact number of RDF triples into semantic reservoir [19]. These advantages become more useful, when the precise load onto each of the listed path is calculated by means of individual cognitive nodes' load. We arrange 6 random paths with the help of simulation environment based on ant colony optimization approach and calculates that path ' $A$ ' consumes least average load percentile than other cognitive network paths as shown in Fig. 9. The reason of such a low load consumption refers to the involvement of lower HOP count number in processing the RDF triples on cognitive network 


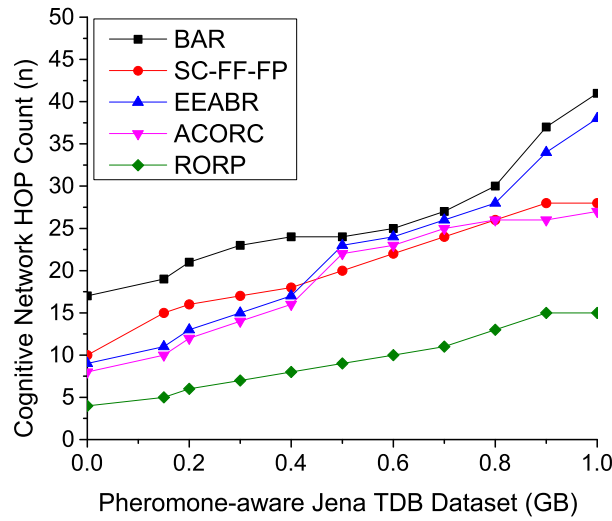

Fig. 12: HOP count analysis with multiple path optimizers

as shown in Fig. 10.

5) Comparative analysis: The comparative analysis includes BAR, SC-FF-FP, EEABR and ACORC [20] implementations onto pheromone-aware RDF processing in a cognitive network of smart grid. The basic ant routing (BAR) is a fundamental routing approach that records limited range of footsteps with a default payload segment less than 200 footsteps. The SC-FF-FP, EEABR and ACORC also observes a routing table that has limited capacity with an average buffer flush of 1000 footsteps. In this way, RORP is far better than discussed approaches because of its flexible range solely dependent onto indexing range of semantic reservoir. Secondly, all of the discussed approaches believe that pheromone evaporates with a less or more time-stamp according to the functional performance of ant colony approach [12], where as, RORP does not believe in evaporation of pheromone and acquires a consistent broad range of pheromone-value that helps to analyze the path load and HOP count accuracy in transmitting RDF triples onto cognitive network. The third important aspect which specifically reflects a prominent difference among all the discussed approaches and can be known as decisive metrics to evaluate RORP with other approaches is the extraction of cognitive node load and optimal path through persistent dataset into semantic reservoir. Keeping these factors and metrics into consideration, we compare RORP with these existing ant colony approaches [20] and observes an optimal load throughout the cognitive network than other existing approaches. Also, we get less number of HOP counts as compared to previous approaches onto the cognitive network of smart grid as shown in Fig. 11 and Fig. 12.

\section{CONClusion}

In this paper, we propose RORP that monitors load of cognitive nodes through pheromone-aware RDF analytics in the smart grid. The proposed approach applies a novel enhancement of payload segment into three sub-payloads so that each tuple such as subject, predicate and object can be transmitted efficiently in the cognitive node environment. Also, the proposed approach adds pheromone onto the RDF payloads so that cognitive process can analyze the node workload and evaluate optimal processing path with least HOP count in the smart grid. The evaluation results show that RORP effectively addresses the RDF data transmission and identifies optimal load path with least HOP count in the cognitive network of the smart grid.

\section{REFERENCES}

[1] S. Suthaharan, "Big data classification", ACM SIGMETRICS Performance Evaluation Review, vol. 41, no. 4, pp. 70-73, 2014.

[2] E. Fadel, M. Faheem, V. Gungor, L. Nassef, N. Akkari, M. Malik, S. Almasri and I. Akyildiz, "Spectrum-aware bio-inspired routing in cognitive radio sensor networks for smart grid applications", Computer Communications, vol. 101, pp. 106-120, 2017.

[3] I. F. Siddiqui, S. U. J. Lee, A. Abbas and A. K. Bashir, "Optimizing Lifespan and Energy Consumption by Smart Meters in Green-CloudBased Smart Grids," in IEEE Access, vol. 5, pp. 20934-20945, 2017.

[4] I. F. Siddiqui, A. Abbas and S. U. J. Lee, "A HIDDEN MARKOV MODEL TO PREDICT HOT SOCKET ISSUE IN SMART GRID", Journal of Theoretical and Applied Information Technology vol.94.2, pp.408, 2016.

[5] S. Baidya and M. Levorato, "Content-Aware Cognitive Interference Control for Urban IoT Systems", IEEE Transactions on Cognitive Communications and Networking, pp. 1-1, 2018.

[6] D. Goel, S. Chaudhury and H. Ghosh, "An IoT approach for contextaware smart traffic management using ontology", Proceedings of the International Conference on Web Intelligence - WI '17, 2017.

[7] Y. Zhang, J. Lopez, J. Tang, Y. C. Liang and X. Zhang, "Wireless Big Data: Technologies and Applications," IEEE Wireless Communications, vol. 25, no. 1, pp. 10-11, February 2018.

[8] S. Kafaie, Y. Chen, O. A. Dobre and M. H. Ahmed, ”Joint Inter-flow Network Coding and Opportunistic Routing in Multi-hop Wireless Mesh Networks: A Comprehensive Survey", IEEE Communications Surveys \& Tutorials, pp. 1-1, 2018

[9] J. Wan, B. Chen, M. Imran, F. Tao, D. Li, C. Liu and S. Ahmad, "Toward Dynamic Resources Management for IoT-Based Manufacturing", IEEE Communications Magazine, vol. 56, no. 2, pp. 52-59, 2018.

[10] K. Han, Y. Ko and J. Kim, "A novel gradient approach for efficient data dissemination in wireless sensor networks", IEEE 60th Vehicular Technology Conference, 2004.

[11] S. Chiang, C. Huang and K. Chang, "A Minimum Hop Routing Protocol for Home Security Systems Using Wireless Sensor Networks", IEEE Transactions on Consumer Electronics, vol. 53, no. 4, pp. 1483-1489, 2007.

[12] J. Ho, H. Shih, B. Liao, and S. Chu, "A ladder diffusion algorithm using ant colony optimization for wireless sensor networks", Information Sciences, vol. 192, pp. 204-212, 2012.

[13] T. Du, S. Qu, Q. Wang, "An Energy Aware Ladder Diffusion Routing Algorithm for WSNs", Lecture Notes on Information Theory, vol. 2, no. 1, pp. 1-6, 2014.

[14] M. Koubarakis, K. Bereta, G. Papadakis, D. Savva and G. Stamoulis, "Big, Linked Geospatial Data and Its Applications in Earth Observation", IEEE Internet Computing, vol. 21, no. 4, pp. 87-91, 2017.

[15] R. Moghaddass and J. Wang, "A Hierarchical Framework for Smart Grid Anomaly Detection Using Large-Scale Smart Meter Data", IEEE Transactions on Smart Grid, pp. 1-1, 2017.

[16] T. Weibel, "Smart - UMass Trace Repository", Traces.cs.umass.edu, 2018. [Online]. Available: http://traces.cs.umass.edu/index.php/Smart/Smart. [Accessed: 06Apr- 2018].

[17] DM4T, "frictionlessdata/pilot-dm4t", GitHub, 2018. [Online]. Available: https://github.com/frictionlessdata/pilot-dm4t. [Accessed: 06- Apr2018].

[18] L. Suzuki, "Smart Cities IoT: Enablers and Technology Road Map", Smart City Networks, pp. 167-190, 2017.

[19] A. Iyengar, "Supporting Data Analytics Applications Which Utilize Cognitive Services", IEEE 37th International Conference on Distributed Computing Systems (ICDCS), 2017.

[20] Z. Zhou, F. Xiong, C. Xu, Y. He and S. Mumtaz, "Energy-Efficient Vehicular Heterogeneous Networks for Green Cities," IEEE Transactions on Industrial Informatics, vol. 14, no. 4, pp. 1522-1531, April 2018. 